\title{
Formulation and evaluation of Metformin Hydrochloride-loaded Curcumin-Lycopene Niosomes
}

\author{
A. Katrolia ${ }^{1}$. S. B. Chauhan ${ }^{1}$-V. K. Shukla ${ }^{1}$
}

Received: 8 August 2019 / Accepted: 22 November 2019 / Published online: 28 November 2019

(c) Springer Nature Switzerland AG 2019

\begin{abstract}
Curcumin and lycopene, both are naturally occurring potent antioxidants which can be used in the treatment of oxidative stress. The biological sources to obtain curcumin and lycopene are curcuma longa and Solanum lycopersicum, respectively. The aim of this research study is to develop curcumin-lycopene conjugate-based biguanide niosomes. Span 60 as non-ionic surfactant and cholesterol as a stabilizer were used for the preparation of metformin hydrochloride-loaded niosomes by the ethanol injection method. This research dealt with the composition, formulation, evaluation and optimization of niosomes. The particle size and polydispersity index were found to be in the range from 139.6 to 509 and 0.152 to $0.541 \mathrm{~nm}$, respectively. The entrapment efficiency and drug loading capacity were observed in the range of 40.43-79.02 and 26.954 to $78.17 \%$, respectively. The in vitro drug release studies showed the sustained release of drug in all formulations up to the period of $24 \mathrm{~h}$. Kinetic analysis of in vitro drug release studies showed that when the ratio of Span 60 and cholesterol is 1:1.5 and 1:2, the formulations followed first-order kinetics. When the ratio changed to 1:1, the formulation followed the Korsmeyer-Peppas model and finally at the ratio of 1.5:1 and 2:1, formulations followed the Higuchi kinetics and Hixson-Crowell kinetics, respectively. Formulation S2 is concluded as an optimized formulation on the basis of all evaluation parameters.
\end{abstract}

Keywords Curcumin-lycopene conjugate $\cdot$ Metformin hydrochloride $\cdot$ Niosomes $\cdot$ Ethanol injection method $\cdot$ Span 60

\section{Introduction}

Niosomes are defined as vesicular structures composed of non-ionic surfactant and cholesterol. They are known for the entrapment of hydrophobic as well as hydrophilic drugs [1] (Fig. 1).

Advantages of Niosomes:

1. Niosomes provide better patient compliance.

2. They are capable of entrapping hydrophilic, lipophilic as well as amphiphilic drugs.

3. They provide controlled and sustained release of drugs.
4. Oral bioavailability of the drug can be improved by using niosomes [1].

5. They are biodegradable, biocompatible and nonimmunogenic to the body.

6. They provide targeting of drugs to various organs.

7. They provide enhanced stability of entrapped drugs [3].

Curcumin is an active constituent of Curcuma longa and is extracted from its rhizomes. Chemically, CUR (diferuloylmethane) is 1,7-bis(4-hydroxy-3-methoxy-phenyl)-hepta1,6-diene-3,5-dione and it is represented as $\mathrm{C}_{21} \mathrm{H}_{20} \mathrm{O}_{6}$ [4]. Oral route is the main route of administration of turmeric preparations. But due many barriers such as its

A. Katrolia, architakatrolia95@gmail.com; S. B. Chauhan, shikha.pharma@gmail.com; V. K. Shukla, vikeshg2002@gmail.com| 1 Amity Institute of Pharmacy, Amity University, Noida, Uttar Pradesh, India. 


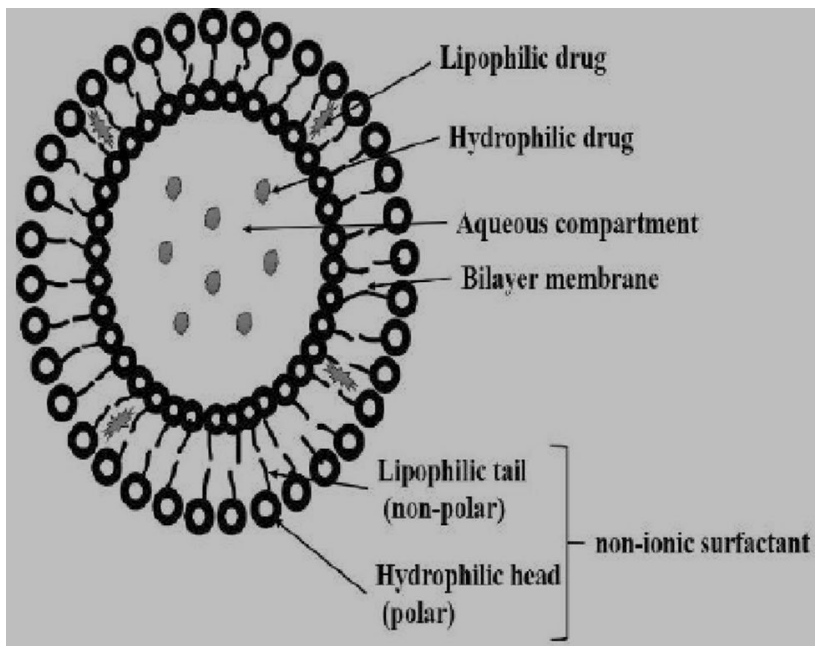

Fig. 1 Structure of niosome [2]

hydrophobicity at physiological $\mathrm{pH}$, limited absorption, rapid metabolism and excretion, it has low bioavailability. To overcome the problems of poor solubility and low bioavailability, nanoparticle-based drug delivery system has been suggested [5].

Lycopene is a polyene, unsaturated hydrocarbon carotenoid. It has a molecular weight of $536.888 \mathrm{~g} / \mathrm{mol}$. It is an acyclic open-chain unsaturated carotenoid having 13 double bonds, 11 of them are conjugated double bonds arranged in a linear array. Antioxidant property of lycopene is associated with its extended system of conjugated double bonds.

In this research, curcumin-lycopene conjugate was prepared to get the synergistic effect of their antioxidant activity. Metformin hydrochloride that is the choice of drug was loaded in the conjugate in the form of niosomes by using ethanol injection method. This was done in order to obtain a targeted drug delivery system and to improve the oral bioavailability of the drug.

\section{Materials and methods}

\subsection{Materials}

Cholesterol, Span 60 (Central Drug House, New Delhi), ethanol (Changshu Hongsheng Fine Chemicals Co. Ltd.), curcumin (Central Drug House, New Delhi), lycopene (Medcure Pharma, Baddi), dichloromethane (S D Fine Chem Limited, Mumbai) and metformin hydrochloride (Accent Pharma, SIDCO, Jammu) were used in the study.

\subsection{Preparation of niosomes}

Ethanol injection method [6] was used for the preparation of niosomes. Firstly, curcumin and lycopene in specified amounts were dissolved in $10 \mathrm{~mL}$ of ethanol and dichloromethane, respectively. Both the solutions were mixed by using magnetic stirrer. Then, the cholesterol and Span 60 were added in the ratio as specified in Table 1 into above solution and stirred until a homogenous mixture was obtained. This will form the lipid phase (A) of the formulation. Aqueous phase (B) was prepared by dissolving $50 \mathrm{mg}$ of drug in $100 \mathrm{~mL}$ distilled water. Finally, the solution $A$ was added dropwise into solution $B$, maintained at $60^{\circ} \mathrm{C}$ with continuous stirring at $1750 \mathrm{rpm}$. The mixture was stirred until the organic solvents get evaporated completely.

\subsection{Characterizations of niosomes}

\subsubsection{Morphology}

The prepared niosomal formulation was studied under the microscope for morphological evaluation. A drop of preparation was poured on a glass slide and placed under the pre-adjusted optical microscope. The morphology was then studied carefully [7].
Table 1 Formula for different batches of niosome

\begin{tabular}{lllllll}
\hline Ingredients & MH $(\mathrm{mg})$ & CUR $(\mathrm{mg})$ & $\begin{array}{l}\text { Lycopene } \\
(\mathrm{mg})\end{array}$ & Span 60 $(\mathrm{mg})$ & $\begin{array}{l}\text { Cholesterol } \\
(\mathrm{mg})\end{array}$ & $\begin{array}{l}\text { Stirring } \\
\text { speed } \\
(\mathrm{rpm})\end{array}$ \\
\hline S1 & & & & & & \\
\hline S2 & 50 & 25 & 25 & 50 & 50 & 1750 \\
S3 & 50 & 25 & 25 & 75 & 50 & 1750 \\
S4 & 50 & 25 & 25 & 50 & 75 & 2000 \\
S5 & 50 & 25 & 25 & 50 & 100 & 1750 \\
\hline
\end{tabular}

$\mathrm{MH}$ metformin hydrochloride, CUR curcumin 


\subsubsection{Particle Size and polydispersity index (Pdl)}

Dynamic light scattering technique was used to determine the particle size and Pdl of formulated niosomes. The samples were firstly diluted with the help of distilled water and then were filled in the cuvettes. The cuvettes were placed in the instrument (Zetasizer Nano ZS-90; Malvern Instruments Ltd. UK), and finally, particle size and Pdl were determined at the angle and temperature of $90^{\circ}$ and $25^{\circ}$, respectively $[7,8]$.

\subsubsection{Entrapment efficiency (\%EE)}

$10 \mathrm{~mL}$ of prepared formulation was taken and centrifuged at $7000 \mathrm{rpm}$ at $-4^{\circ} \mathrm{C}$ for $1 \mathrm{~h}$ to separate the free drug from the niosomes. The supernatant was then collected and diluted properly with phosphate buffer saline (PBS) at $\mathrm{pH}$ 6.4. Finally, the dilutions were run at $232 \mathrm{~nm}$ using UVspectrophotometer to determine the amount of free drug. The amount of entrapped metformin was calculated by subtracting the amount of free drug from the total drug present in the $10 \mathrm{~mL}$ of niosomal preparation $[9,10]$.

$\% E E=\left(d_{t}-d_{f} / d_{t}\right) \times 100$

Here, $d_{t}=$ total amount of drug; $d_{f}=$ amount of free drug

\subsubsection{Drug loading capacity (\%DL)}

It can be calculated as per formula.

$\% D L=\frac{\text { Amount of total entrapped drug }}{\text { Total weight of nanoparticles }} * 100$

\subsubsection{In vitro drug release studies}

An amount of niosomal formulation, equivalent to $2.0 \mathrm{mg}$ of drug, was calculated and filled in the dialysis bag. A beaker containing $100 \mathrm{~mL}$ of PBS at $\mathrm{pH} 6.4$ was placed over a magnetic stirrer at $100 \mathrm{rpm}$, and the temperature was maintained at $37 \pm 1{ }^{\circ} \mathrm{C}$. Then, the bottom of the dialysis bag was dipped into the beaker. Finally, aliquots $(5 \mathrm{~mL})$ were withdrawn periodically from the beaker and the equal volume of PBS was added to maintain the sink conditions. The test was continued for $24 \mathrm{~h}$. The withdrawn aliquots were then subjected to UV-spectrophotometry analysis at $232 \mathrm{~nm}$ to determine the concentration of drug $[11,12]$.

\subsubsection{Kinetic analysis of in vitro release profiles}

To investigate the mechanism of drug release from prepared niosomes, the release data were analysed by using different mathematical models as follows [13].
Zero order A graph of \%CDR against time was plotted to study the release kinetics.

First order A graph of log \%DR against time was plotted to study the release kinetics.

Higuchi model A graph of \%CDR against SQRT was plotted to study the release kinetics.

Korsmeyer-Peppas model A graph log \%CDR against log time was plotted to study the release kinetics.

Hixson-Crowell model A graph of CBRT \%DR against time was plotted to study the release kinetics [14].

\subsubsection{Scanning electron microscopy}

The surface morphology of the formulation was studied by using SEM. The prepared niosomal formulation was deep frozen and lyophilized prior to SEM. A double-sided conducting tape was taken, and the lyophilized sample was spread over it. Then, the coating was done with gold by using gold sputter under vacuum condition but in the presence of argon gas at $50 \mathrm{~mA}$ for $100 \mathrm{~s}$. Finally, the sample was analysed under the microscope and the images were taken (Fig. 2).

\subsubsection{Physical stability testing}

The physical stability of niosomes was studied by leakage of the drug from niosomal vesicles in the innate prepared form that is dispersion stored at room temperature as well as under refrigeration. The optimized formulation was taken in the dispersion form and stored in sealed vessels at $2-8{ }^{\circ} \mathrm{C}$ and at room temperature for a period of 90 days. The samples were withdrawn at regular interval of time, i.e. $0,15,30,60$ and 90 days and then evaluated for percentage drug retained [15].

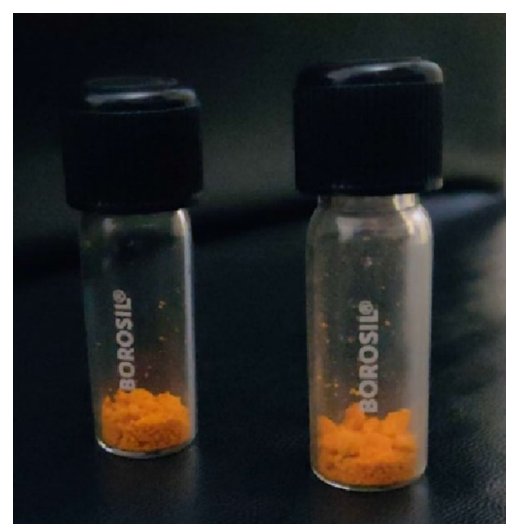

Fig. 2 Lyophilized formulation 


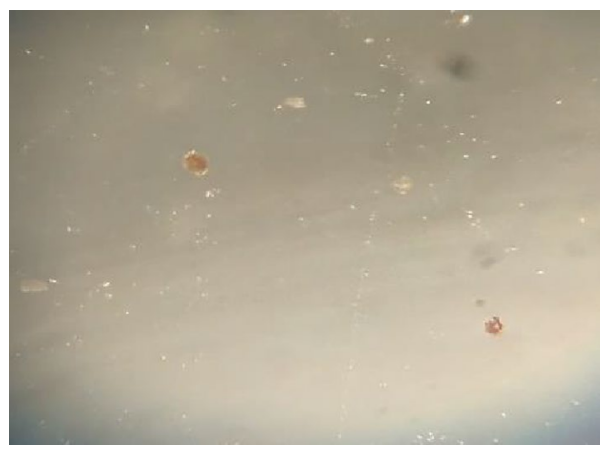

Fig. $3 \mathrm{MH}$ niosomes under microscope

Table 2 Particle size and polydispersity index of niosomes

\begin{tabular}{lll}
\hline Formulations & Particle size $(\mathrm{nm})$ & Pdl \\
\hline S2 & 395.9 & 0.337 \\
S4 & 440 & 0.454 \\
S6 & 253.6 & 0.152 \\
S8 & 139.6 & 0.223 \\
S9 & 509 & 0.541 \\
\hline
\end{tabular}

\section{Results and discussions}

\subsection{Morphology}

The microscopic view as depicted in Fig. 3 of the drug loaded niosomes shows the niosomes formed were spherical in shape.

\subsection{Particle size and PdI}

The particle size and Pdl was found to be in the range of $139.6-509 \mathrm{~nm}$ and 0.152-0.541, respectively, as shown in Table 2.

\subsection{Entrapment efficiency and drug loading capacity}

The entrapment efficiency and drug loading capacity were observed in the range of $40.43-79.02$ and $26.954-78.17 \%$, respectively, as shown in Table 3.

\subsection{In vitro drug release studies}

Figure 4 shows the drug release profiles in PBS at $\mathrm{pH}$ 6.4. Formulation S4 showed the minimum release of $22.221 \%$, whereas formulation $\mathrm{S} 2$ showed the maximum release of $70.607 \%$ over a period of $24 \mathrm{~h}$. It is clear from the graph that the $\mathrm{MH}$ release in vitro has undergone rapid initial burst followed by slow sustained release. The rapid release
Table 3 Entrapment efficiency and drug loading capacity of niosomes

\begin{tabular}{lll}
\hline Formulations & $\begin{array}{l}\text { Percentage entrapment } \\
\text { efficiency (\%EE) }\end{array}$ & $\begin{array}{l}\text { Drug loading } \\
\text { capacity (\%DL) }\end{array}$ \\
\hline S2 & 69.73 & 34.865 \\
S4 & 79.02 & 78.17 \\
S6 & 40.43 & 26.954 \\
S8 & 62.77 & 31.385 \\
S9 & 48.21 & 48.21 \\
\hline
\end{tabular}

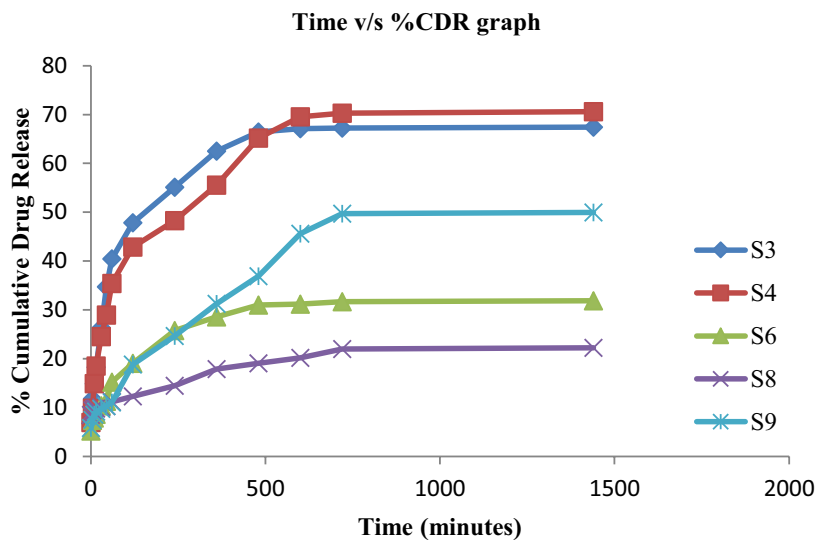

Fig. 4 Time vs \% CDR graph

may be due to drug incorporated in fatty acid chains of lipid bilayers. This led to the rapid release of MH upon dispersing vesicles in buffer until reaching equilibrium [16].

\subsection{Kinetic analysis of in vitro release studies}

See Tables 4 and 5

\subsection{Scanning electron microscopy}

See Figs. 5, 6, 7, 8 and 9.

\subsection{Physical stability testing}

The optimized formulation S2 was investigated for physical stability studies. The results of this investigation showed that after the period of 30 days at room temperature, the percentage drug entrapped in niosomes was found to be $99.93 \%$, whereas at $2-8{ }^{\circ} \mathrm{C}$, it was $99.98 \%$. At the end of the study, i.e. after 90 days, the percentage drug entrapped was found to be $99.6 \%$ and $99.97 \%$ at room temperature and at $2-8{ }^{\circ} \mathrm{C}$, respectively.

On the basis of derived particle size, polydispersity index, entrapment efficiency, drug loading capacity, in vitro drug release studies, kinetic analysis of drug 
Table 4 Statistical kinetic values for different formulations

\begin{tabular}{llllll}
\hline Batch & Zero-order model & First-order model & Higuchi model & $\begin{array}{l}\text { Korsmeyer- } \\
\text { Peppas model }\end{array}$ & $\begin{array}{l}\text { Hixson- } \\
\text { Crowell } \\
\text { model }\end{array}$ \\
\hline S1 & 0.8152 & 0.9137 & 0.9521 & 0.9849 & 0.8659 \\
S2 & 0.9241 & 0.979 & 0.981 & 0.9351 & 0.968 \\
S3 & 0.9783 & 0.9794 & 0.9472 & 0.8116 & 0.9791 \\
S4 & 0.9824 & 0.9845 & 0.9543 & 0.7983 & 0.9839 \\
S5 & 0.986 & 0.978 & 0.9431 & 0.843 & 0.99 \\
\hline
\end{tabular}

Table 5 Kinetic model followed by different formulations

\begin{tabular}{llll}
\hline Batch & Span 60:CH & Kinetic model & Remarks \\
\hline S1 & $1: 1$ & Korsmeyer-Peppas model & Describes the drug release from a polymeric system [13] \\
S2 & $1.5: 1$ & Higuchi model & Describes the release of drug from the insoluble matrix [13] \\
S3 & $1: 1.5$ & First order & Describes the water soluble drug dissolution in porous matrices [14] \\
S4 & $1: 2$ & First order & Describes the water soluble drug dissolution in porous matrices \\
S5 & $2: 1$ & Hixson-Crowell model & $\begin{array}{l}\text { Change in surface area and diameter of the particles describes the } \\
\text { release from the system [14] }\end{array}$ \\
\hline
\end{tabular}

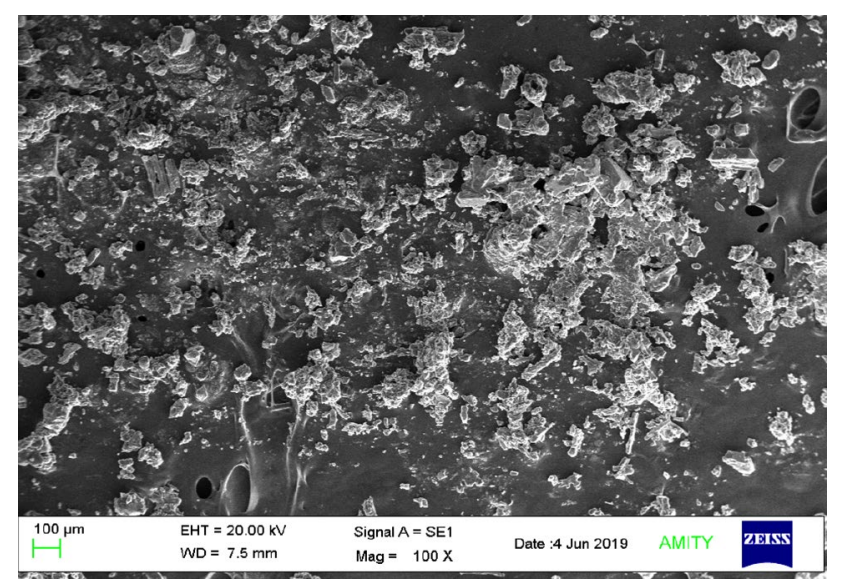

Fig. 5 Formulation at $\times 100$ magnification

release studies and scanning electron microscopy, formulation $\mathrm{S} 2$ are selected as an optimized formulation. This is due to the fact that $\mathrm{S} 2$ gives the desired particle size and entrapment efficiency. It also provides the sustained release of metformin hydrochloride over the period of $24 \mathrm{~h}$ with maximum drug release of $70.607 \%$. Formulation S2 follows Higuchi model (plots show the highest linearity). Higuchi kinetics explains that the drug diffuses at a slower rate as the distance for diffusion increases, referred to the square root kinetics. In niosomes, liquid penetrates the matrix and dissolves the drug, which then diffuses into the exterior liquid. Higuchi tried to relate the drug release rate to the physical constants based on simple law of diffusion (Fick's first law). Also, the results showed that the niosomes were more stable under refrigeration, i.e. at $2-8{ }^{\circ} \mathrm{C}$. The

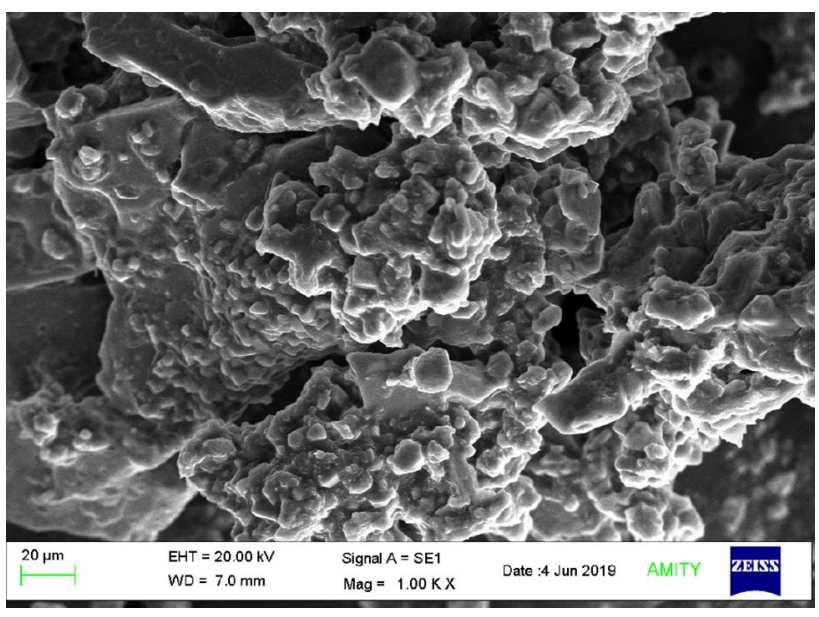

Fig. 6 Formulation at $\times 1000$ magnification

percentage drug retained in the niosomes after the period of 90 days under refrigeration was found to be $99.97 \%$.

\section{Conclusion}

In this research work, five formulations were prepared at different ratios of Span 60 and cholesterol by using ethanol injection method. After that, various evaluation tests were performed to determine particle size, polydispersity index, percentage entrapment efficiency, drug loading capacity and in vitro drug release. Kinetic models were also studied for different formulations. From the obtained results, it was concluded that S2 is an optimized formulation with desired particle size and entrapment efficiency of 


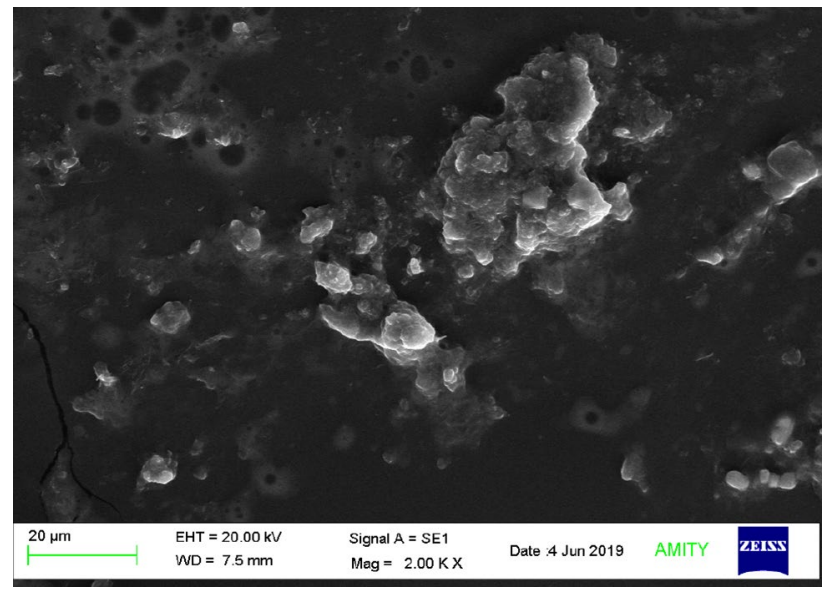

Fig. 7 Formulation at $\times 2000$ magnification

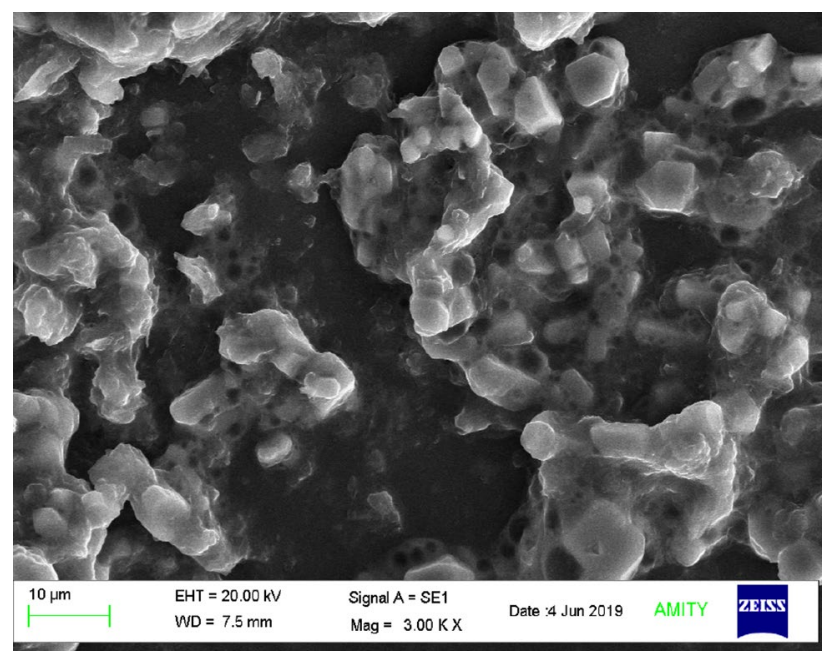

Fig. 8 Formulation at $\times 3000$ magnification

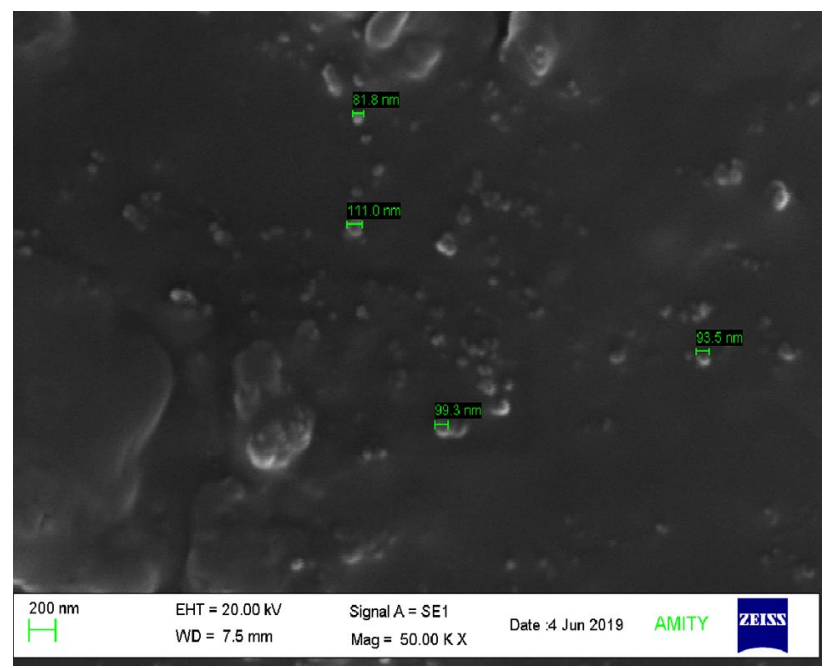

Fig. 9 Formulation at $\times 50,000$ magnification
$440 \mathrm{~nm}$ and $79.02 \%$, respectively. It also showed the maximum release of $70.607 \%$ over a period of $24 \mathrm{~h}$. Finally, formulation S2 followed Higuchi model kinetics, the reason being that plots shows the highest linearity and showed the high physical stability under refrigeration conditions by retaining $99.97 \%$ drug after a period of 90 days.

\section{References}

1. Rajera R, Nagpal K, Singh SK, Mishra DN (2011) Niosomes: a controlled and novel drug delivary system. Biol Pharm Bull 34(7):945-953

2. Satish R, Yashashri I, Bhushan R (2018) Niosomes: a non-Ionic surfactants based vesicles as a carriers for drug delivery. Int J Pharm Sci Rev Res 198-213

3. Satish R, Yashashri I, Bhushan R, Ashish J (2018) Niosomes: a nonionic surfactant based vesicles as a carriers for drug delivery. Int J Pharm Sci Res 29:198-213

4. Amalraj A, Pius A, Gopi S, Gopi S (2017) Biological activities of curcuminoids, other biomolecules from turmeric and their derivatives-a review. J Tradit Complement Med 7(2):205-233

5. Essa EA (2014) Effect of formulation and processing variables on the particle size of sorbitan monopalmitate niosomes. Asian J Pharm 4:289

6. Bhaskaran S, Lakshmi PK (2009) Comparative evaluation of niosome formulations prepared by different techniques. Acta Pharm Sci 51(1):27-32

7. Hasan AA, Hafez M (2013) Formulation and evaluation of metformin hydrochloride-loaded niosomes as controlled. Drug Deliv 20:120-126

8. Seleci DA, Muharrem S, Johanna-Gabriela W, Frank S, Thomas S (2016) Niosomes as nanoparticular drug carriers: fundamentals and recent applications. J Nanomater 2016:1-13

9. Bhama SK, Rakhi K, Lakshmi PS, Vasudev DT, Nair SC (2017) Formulation and evaluation of niosomal suspension of Cefixime. Asian J Pharm Clin Res 10(5):194-201

10. D. Pando, G. Gutierrez, J. Coca, C. Pazos, (2013) Preparation and characterization of niosomes containing resveratrol, Journal of Food Engineering, page no. 227-234

11. Kandasamy R, Veintramuthu S (2010) Formulation and Optimization of zidovudine niosomes. AAPS PharmSciTech 11(3):1119-1127

12. Momoh MA, Kenechukwu FC, Attama AA (2013) Formulation and evaluation of novel solid lipid microparticles as a sustained release system for the delivery of metformin hydrochloride. Drug Deliv 20(3-4):102-111

13. Singhvi Gautam, Singh Mahaveer (2011) Review: in vitro drug release characterization model, Int J Pharm Stud Res II(1):77-84

14. Dash Suvakanta, Murthy Padala Narasimha, Nath Lilakanta, Chowdhury Prasanta (2010) Kinetic modeling on drug release from controlled drug delivery systems. Acta Pol Pharm Drug Res 67(3):217-223

15. Ajay S, Jolly P, Rajesh P (2008) Preparation, characterization, optimization, and stability studies of aceclofenac proniosomes. Iran J Pharm Res 7(4):237-246

16. El-Ridy MS, Yehia SA, Mohsen AM, El-Awdanc SA, Darwish AB (2017) Preparation, characterization and in-vivo evaluation of span 60-lornoxicam niosomes. Res J Pharm Biol Chem Sci 398:398-411

Publisher's Note Springer Nature remains neutral with regard to jurisdictional claims in published maps and institutional affiliations. 